Pacific Journal of Mathematic 


\section{ASYMPTOTIC LOWER BOUNDS FOR THE FUNDAMENTAL FREQUENCY OF CONVEX MEMBRANES}

George E. Forsythe

1. Introduction. Let the bounded, simply connected, open region $R$ of the $(x, y)$-plane have the boundary curve $C$. If a uniform ideal elastic membrane of unit density is uniformly stretched upon $C$ with unit tension across each unit length, then $\lambda$, the square of the fundamental frequency, satisfies the conditions (subscripts denote differentiation)

$$
\left\{\begin{aligned}
\Delta u & \equiv u_{x x}+u_{y y}=-\lambda u \text { in } R, \\
\lambda & =\text { minimum }
\end{aligned}\right.
$$

with the boundary condition

$$
u(x, y)=0 \text { on } C .
$$

Variational methods of the Rayleigh-Ritz type are frequently used to approximate $\lambda$. They always yield upper bounds for $\lambda$, and the upper bounds can be made arbitrarily close.

Another common practical method of approximating $\lambda$ is to calculate the least eigenvalue $\lambda_{h}$ of a suitably chosen finite-difference operator $\Delta_{h}$ over a network with small mesh width $h$. For one choice of $\Delta_{h}$ it was shown by Courant, Friedrichs, and Lewy [3, p. 57] without details that $\lambda_{h} \rightarrow \lambda$ as $h \rightarrow 0$. For convex regions $R$ of a special polygonal form the author has shown [4] that a special case of (11) below is valid for a common choice of $\Delta_{h}$, and hence that $\lambda_{h}$ is asymptotically a lower bound for $\lambda$ as $h \rightarrow 0$. For an unusual finite-difference approximation to problem (1) when $R$ is the union of squares of the network, Polya [12] has found that $\lambda_{h}>\lambda$ for all $h$, and also for the higher eigenvalues. The author knows of no other study of the sign or order of decrease of $\lambda-\lambda_{h}$ to 0 .

In the present paper the investigation of [4] is extended to a much wider class of regions: those with piecewise analytic boundary curves and convex corners. The new theorems are stated and proved in $\S \S 3$ and 4. Theorem 2 contains the theorem of [4] as a special case. Lemmas used in the proof of Theorem 1 are given in $\S 5$. Identity (31) of Lemma 7 is interesting in itself.

Received December 2, 1953. Presented to the American Mathematical Society September 4, 1953. The preparation of this paper was sponsored in part by the Office of Naval Research, U.S.N. 
When $C$ is no longer made up of line segments of the network, it is necessary when using finite-difference methods either to move $C$ or to alter $\Delta_{h}$ near the boundary. The latter procedure is potentially more accurate, and has been adopted in deriving the rather delicate results proved below. The definition of $\Delta_{h}$ given in $\S 2$ is a self-adjoint modification of Mikeladze's approximation [10;11], and is believed to be new. The cruder approximations to $\triangle$ near $C$ proposed by Collatz in 1933 and expounded in [2, p. 357], while easier to compute in practice, appear to introduce an unmanageable term $O\left(h^{2}\right)$ into (19). It is therefore doubted that Theorem 2 would remain valid for these cruder operators.

The technique of the present paper could be applied to study the asymptotic behavior of $\lambda_{h}$ also for other difference approximations to $A$ in the interior of $R$-for example, for those associated with a triangular net $[2$, p. 367].

It is not clear that one could revise the argument of the paper to prove an inequality of the type

$$
\frac{\lambda}{\lambda_{h}} \leq 1+b h^{2}+o\left(h^{2}\right) \text {. }
$$

2. Definitions. Assume the bounded, simply connected, open region $R$ to have a closed boundary curve $C: x(s)+i y(s)\left(0 \leq s \leq s_{m}\right)$ which is piecewise analytic. That is, $x(s)$ and $y(s)$ are real analytic functions of the arc length $s$ of $C$ in each of a finite number $m$ of closed intervals

$$
0=s_{0} \leq s \leq s_{1}, \quad s_{1} \leq s \leq s_{2}, \quad \cdots, \quad s_{m-1} \leq s \leq s_{m} .
$$

Moreover, we demand that the corners of $C$ be convex; that is, at any point $x\left(s_{j}\right)+i y\left(s_{j}\right) \quad(0 \leq j<m)$ where distinct analytic curves meet, the interior angle of $C$ must be less than $\pi$.

For $h>0$, let a net consist of the lines $x=\mu h, y=\nu h \quad(\mu, \nu=0, \pm 1$, $\pm 2, \cdots)$. The points $(\mu h, \nu h)$ in $R$ are the interior nodes $R_{h}$ of the net. The boundary nodes $C_{h}$ of the net consist of (i) all points $(\mu h, \nu h)$ on $C$, and (ii) all isolated points of intersection of the net with $C$. Thus each node $(\mu h, \nu h)$ of $R_{h}$ has two neighboring nodes in $R_{h} \cup C_{h}$ on the line $x=\mu h$, and two in $R_{h} \cup C_{h}$ on the line $y=\nu h$. Moreover, each node in $C_{h}$ has at least one neighbor in $R_{h} \cup C_{h}$.

We now move toward a definition of the difference operator $\Delta_{h}$. Let. us denote the neighboring nodes of the node

$$
(x, y) \text { of } R_{h} \text { by }\left(x-h_{1}, y\right),\left(x+h_{2}, y\right),\left(x, y-h_{3}\right) \text {, and }\left(x, y+h_{4}\right) \text {, }
$$

where $0<h_{i} \leq h$ for $i=1,2,3,4$. For nodes remote from $C_{h}$, all $h_{i}=h$. Let $v$ be any net function defined on the nodes of $R_{h} \cup C_{h}$, vanishing 
on $C_{h}$. Define $D_{x}^{(h)} v$ as the (constant) second derivative of the quadratic polynomial function of $x$ assuming the three values $v\left(x-h_{1}, y\right), v(x, y)$, and $v\left(x+h_{3}, y\right)$. That is,

$$
D_{x}^{(h)} v(x, y)=c_{h_{1}+h_{2}}^{2}\left[\begin{array}{cc}
v\left(x+h_{2}, y\right)-v(x, y) \\
h_{2}
\end{array}-v(x, y)-v\left(x-h_{1}, y\right)\right] .
$$

Also, $D_{y}^{(h)} v(x, y)$ is defined analogously. We next define

$$
\begin{aligned}
& \Delta^{(h)} v(x, y)=D_{x}^{(h)} v(x, y)+D_{y}^{(h)} v(x, y) \\
& =-\left(\begin{array}{c}
2 \\
h_{1} h_{2}
\end{array}+\begin{array}{c}
2 \\
h_{3} h_{4}
\end{array}\right) v(x, y) \\
& +\frac{2}{h_{1}\left(h_{1}+h_{2}\right)} v\left(x-h_{1}, y\right)+\frac{2}{h_{2}\left(h_{1}+h_{2}\right)} v\left(x+h_{2}, y\right) \\
& +\frac{2}{h_{3}\left(h_{3}+h_{4}\right)} v\left(x, y-h_{3}\right)+\frac{2}{h_{4}\left(h_{3}+h_{4}\right)} v\left(x, y+h_{1}\right) \text {. }
\end{aligned}
$$

The operator $\Delta^{(h)}$ is the approximation to $\Delta$ recommended in [10]. It linearly transforms the net function $v$ defined over $R_{h}$ into the net function $\Delta^{(h)} v$, also defined over $R_{h}$. But $J^{(h)}$ is not a self-adjoint linear operator; that is, the matrix $A^{(h)}$ of the linear transformation of $v$ into $\Delta^{(h)} v$ is not symmetric.

We define the matrix $A_{h}$ as the symmetric part of the matrix $A^{(h)}$ :

$$
A_{h}=\frac{1}{2}\left[A^{(h)}+A^{(h) P}\right] \text {, }
$$

where $T$ means transpose. Finally, we define $\Delta_{h}$ to be the self-adjoint linear operator corresponding to $A_{i}$.

The explicit expressions for $\Delta_{h}$ assume 16 different forms, depending on the location of $(x, y)$ with respect to $C_{h}$. Although we shall not need these expressions for the present paper, we describe them briefly. If, in any of the four directions from $(x, y)$, the neighboring node-say $\left(x-h_{1}, y\right)$, for definiteness-is in $R_{h}$, then $h_{1}=h$, and there is another node $\left(x-h-h_{1}{ }^{\prime}, y\right)$ in $R_{h} \cup C_{h}$. Then the term $2 v\left(x-h_{1}, y\right) / h_{1}\left(h_{1}+h_{2}\right)$ of (4) is to be replaced by

$$
\begin{gathered}
h_{1}{ }^{\prime}+2 h+h_{2} \\
\left(h_{1}{ }^{\prime}+h\right) h\left(h+h_{2}\right)
\end{gathered} v(x-h, y) .
$$

For any $(x, y)$, the expression for $\Delta_{l}$ is obtained from (4) by making replacements like (6) corresponding to all neighbors of $(x, y)$ in $R_{l}$.

When $(x, y)$ is more than two nodes away from $C_{l}$, so that all $h_{i}=h_{i}{ }^{\prime}=h$, the values of both $\Delta^{(h)}$ and $\Delta_{h}$ reduce to the familiar form used in [4]: 
( 7 )

$$
\begin{aligned}
& \Delta_{h} v(x, y)=\Delta^{(h)} v(x, y) \\
& \quad=\frac{1}{h^{2}}[v(x-h, y)+v(x+h, y)+v(x, y-h)+v(x, y+h)-4 v(x, y)] .
\end{aligned}
$$

Let $\lambda_{n}$ satisfy the following difference equation for a net function $v$ defined in $R_{h} \cup C_{h}$ :

$$
\left\{\begin{aligned}
\Delta_{h} v & =-\lambda_{h} v \text { in } R_{h} \\
\lambda_{h} & =\text { minimum }
\end{aligned}\right.
$$

where $v$ is extended to satisfy the boundary condition

$$
v=0 \text { on } C_{h} \text {. }
$$

It is readily shown that $\lambda_{h}$ is the minimum over all net functions $v$ satisfying $(8 \mathrm{~b})$ of the quotient

$$
\rho_{h}(v)=\frac{-h^{2} \sum_{R_{h}} v \Delta_{h} v}{h^{2} \sum_{R_{h}} v^{2}} .
$$

(This is simply the minimum principle for a definite quadratic form.) By (5), we can write $\rho_{l}(v)$ in the following equivalent form, simpler to use :

$$
\rho_{h}(v)=\frac{-h^{2} \sum_{R_{h}} v \Delta^{(h)} v}{h^{2} \sum_{R_{h}} v^{2}} .
$$

The reason for not using the least eigenvalue $\mu_{h}$ of $\Delta^{(h)}$ in this investigation is that $\mu_{h}$ does not have the foregoing minimum property and, in fact, might turn out to be complex. On the other hand, it is known [9, p. 27] that $\lambda_{h} \leq \mathscr{R}\left(\mu_{h}\right)$, so that when $\mu_{h}$ is real it could conceivably be a better approximation to $\lambda$ than $\lambda_{h}$ is. The relative magnitude of $\left|\lambda_{h}-\lambda\right|$ to $\left|\mu_{h}-\lambda\right|$ is not known.

3. The results. The following new result will be proved in $\S 4$ :

THEOREM 1. Let $R$ be a bounded, open, simply connected region bounded by a piecewise analytic curve $C$ whose corners are convex in the sense of $\S 2$. Let $\tau$ be the angle between the tangent to $C$ and the $x$ axis. Let $u$ solve problem (1) for $R$, and let $u_{n}$ be the normal derivative of $u$ on $C$. Define $\lambda_{h}$ as in $\S 2$. Let

$$
a=a(R)=\frac{\iint_{R}\left(u_{x x}^{2}+u_{y y}^{2}\right) d x d y+\int_{O} u_{n}^{2} \sin ^{2} 2 \tau d \tau}{12 \iint_{R}\left(u_{x}^{2}+u_{y}^{2}\right) d x d y} .
$$




\section{Then $-\infty<a<\infty$ and, as $h \rightarrow 0$, one has}

$$
\frac{\lambda_{h}}{\lambda} \leq 1-a h^{2}+o\left(h^{2}\right) \quad(h \rightarrow 0) .
$$

In Theorem 1 the quantity a can probably be negative for certain nonconvex $R$, because $d \tau$ in (10) will be negative at some points of $C$. But if $R$ is convex we get a stronger result, as an immediate consequence of Theorem 1.

THEOREM 2. Under the hypotheses of Theorem 1, if $R$ is also convex, then $0<a<\infty$, and there exists $h_{0}>0$ such that $\lambda_{h}<\lambda$ for all $h<h_{0}$.

For the operator $\Delta_{h}$ of $\S 2$ the methods of [3] can undoubtedly be followed to show that $\lambda_{h} \rightarrow \lambda$ as $h \rightarrow 0$; the author has not attempted to carry through the details. When $\lambda_{h} \rightarrow \lambda$ as $h \rightarrow 0$, the lower bounds $\lambda_{n_{0}}$ can be made arbitrarily close by choice of $h_{0}$ sufficiently small. Thus for these $R$ the Rayleigh-Ritz methods and the finite-difference methods (8) are theoretically complementary, and together could confine $\lambda$ to an arbitrarily short interval if one knew an upper bound for $h_{0}$.

The author has not developed an upper bound for $h_{0}$ in Theorem 2, although it would be desirable to do so by estimating the term $o\left(h^{2}\right)$. One could always make an intelligent guess based on the behavior of $\lambda_{h}$ for certain $h$.

The constant $a$ of (10) is the best possible for certain rectangular regions; see [4]. That the corners of $C$ be convex seems essential to the validity of Theorem 1 . Indeed, for one nonconvex polygon some heuristics and an experiment mentioned in [4] make it appear that $\lambda_{h}=\lambda+$ $A h^{4 / 3}+o\left(h^{4 / 3}\right)$, where $A>0$. It would be interesting to know the sign of $a$ for the general case of Theorem 1, or in particular when $C$ is a nonconvex analytic curve.

Corners of angle $\pi$ are frequent in engineering practice, and it would be desirable to know how $\lambda_{h}$ behaves when $R$ has such corners. For such corners Lemma 2 is no longer valid. Lewy [7] provides new tools for an attack on corners of angle $\pi$.

4. Proof of Theorem 1. Let $u$ henceforth be the solution of problem (1) for the fundamental eigenvalue $\lambda$. It is known that

$$
\lambda \iint_{R} u^{2} d x d y=\iint_{R}\left(u_{x}^{2}+u_{y}^{2}\right) d x d y .
$$

The proof of Theorem 1, following [4], consists in setting the values of the function $u$ at the nodes of $R_{h} \cup C_{h}$ into the Rayleigh quotient (9) of problem (8). It will be shown that 


$$
\frac{\rho_{h}(u)}{\lambda}=1-a h^{2}+o\left(h^{2}\right) \quad(h \rightarrow 0) .
$$

Since $\lambda_{h} \leq \rho_{h}(u)$, the theorem follows from (13).

The denominator $h^{2} \sum u^{2}$ of $\rho_{h}(u)$ differs from a Riemann sum for $\iint_{R} u^{2} d x d y$ at most by the terms corresponding to squares or part-squares at the boundary $C$. The total contribution of these terms does not exceed the order of magnitude $L h \max _{R} u^{2}$, where $L$ is the length of $C$. Hence a fortiori

$$
h^{2} \sum_{R_{h}} u^{2}=\iint_{R} u^{2} d x d y+o(1) \quad(h \rightarrow 0) .
$$

Let the nodes of $R_{h}$ be divided into three classes:

$$
\left\{\begin{array}{l}
R_{h}{ }^{1}: \text { those within a distance } h \text { of some corner of } C ; \\
R_{h}{ }^{2}: \text { those not in } R_{h}{ }^{1} \text { but within a distance } h \text { of } C ; \\
R_{h}{ }^{3}: \text { the other nodes of } R_{h} .
\end{array}\right.
$$

Split the numerator of $\rho_{h}(u)$ accordingly:

$$
-h^{2} \sum_{R_{h}} u \Delta^{(h)} u=\sum_{i=1}^{3}\left(-h^{2} \sum_{R_{h} i} u \Delta^{(h)} u\right) \equiv \sum_{i=1}^{3} S_{h}^{i}(u) .
$$

There are a fixed number of corners, not exceeding $m$, and at most two nodes of $R_{h}{ }^{1}$ per corner. Moreover $|\nabla u(x, y)|^{2} \rightarrow 0$ as $(x, y) \rightarrow \mathrm{a}$ corner of $C$, by Lemma 1 in $\S 5$. At any node $(x, y)$ of $R_{h}{ }^{1}$ with neighbors denoted as in (2), we find from (3) that

$$
h^{2}\left|u \Delta^{(h)} u\right| \leq \frac{h^{2}(u-0)}{\min h_{i}} \sum_{i=1}^{4}\left|\frac{u-u_{i}}{h_{i}}\right| \leq 4 h^{2} \max |\nabla u|^{2},
$$

where the $u_{\imath}$ are the values of $u$ at the four neighbors of $(x, y)$, and where the maximum of $|\nabla u|^{2}$ is taken over all points within a distance $2 h$ of some vertex. Hence

$$
\left|S_{h}^{1}(u)\right| \leq 8 m h^{2} \max |\nabla u|^{2}=o\left(h^{2}\right) \quad(h \rightarrow 0) .
$$

Using the notation and assertion of Lemma 3, we have

$$
S_{h}^{2}(u)=-h^{2} \sum_{R_{h}{ }^{2}} u \Delta u-\frac{2 h^{3}}{3} \sum_{R_{h}{ }^{2}} u\left(\theta_{x} u_{x x x}^{\prime}+\theta_{y} u_{y y y}^{\prime \prime}\right) .
$$

Since $u$ satisfies (1a),

$$
-h^{2} \sum_{R_{h^{2}}{ }^{2}} u \Delta u=\lambda h^{2} \sum_{R_{h^{2}}{ }^{2}} u^{2}
$$

By (17), (18), and Lemma 4, 


$$
\left|S_{h}^{2}(u)-\lambda h^{2} \sum_{R_{h^{2}}{ }^{2}} u^{2}\right| \leq \frac{2}{3} h^{3} \sum_{R_{h^{2}}{ }^{2}} u\left(\left|u_{x x x}^{\prime}\right|+\left|u_{y y y}^{\prime \prime}\right|\right)=o\left(h^{2}\right) \quad(h \rightarrow 0) .
$$

Thus

$$
S_{h}^{2}(u)=\lambda h^{2} \sum_{R_{h}^{2}} u^{2}+o\left(h^{2}\right) \quad(h \rightarrow 0)
$$

Similarly, using the notation and assertion of Lemma 5, and by (1a), we have

$$
S_{h}^{3}(u)=\lambda h^{2} \sum_{R_{h}{ }^{3}} u^{2}-\frac{h^{4}}{12} \sum_{R_{h}{ }^{3}} u\left(u_{x x x x}^{\prime}+u_{y y y y}^{\prime \prime}\right)
$$

Now

$$
h^{2} \sum_{R_{h}{ }^{2} \cup R_{h^{3}}{ }^{3}} u^{2}=h^{2} \sum_{R_{h}} u^{2}-h^{2} \sum_{R_{h}{ }^{1}} u^{2}=h^{2} \sum_{R_{h}} u^{2}+o\left(h^{2}\right),
$$

since $u(x, y) \rightarrow 0$ as $(x, y) \rightarrow C$, and since there are at most $2 m$ vertices in $R_{h}{ }^{1}$. Adding (19) and (20), and using (21), we find that

$$
\begin{aligned}
S_{h}^{2}(u)+S_{h}^{3}(u) & =\lambda h^{2} \sum_{R_{h}} u^{2}-\frac{h^{4}}{12} \sum_{R_{h}{ }^{3}} u\left(u u_{x x x x}^{\prime}+u_{y y y y}^{\prime \prime}\right)+o\left(h^{2}\right) \\
& =\lambda h^{2} \sum_{R_{h}} u^{2}-\frac{h^{4}}{12} \iint_{R} u\left(u_{x x x x}+u_{y y y y}\right) d x d y+o\left(h^{2}\right),
\end{aligned}
$$

by Lemma 6 . Adding $S_{h}{ }^{1}(u)$ to the above, and dividing by (14), we find that

$$
\begin{aligned}
\rho_{h}(u) & =\frac{\sum_{i=1}^{3} S_{h}{ }^{i}(u)}{h^{2} \sum_{R_{h}} u^{2}} \\
& =\lambda-\frac{h^{2} \iint_{R} u\left(u_{x x x x}+u_{y y y y}\right) d x d y}{12} \iint_{R} u^{2} d x d y
\end{aligned}
$$

Finally, dividing (22) by $\lambda$, and applying Lemma 7 and (12), one proves (13) and hence Theorem 1.

5. Some lemmas. The following lemmas are basic to the proof of Theorem 1. In all of them $R$ satisfies the conditions stated at the start of $\S 2$, while $u=u(x, y)$ solves problem (1).

LEMMA 1. The function $u$ is an analytic function of $x$ and $y$ in $R \cup C$, except possibly at the corners of $C$. Let $r$ be the distance of $(x, y)$ from a corner $P$ with interior angle $\pi / \alpha, 1<\alpha<\infty$. Then for $m=0,1$, $2, \cdots$, any partial derivative of $u$ of order $m$ has the local representation 


$$
\frac{\partial^{m} u}{\partial x^{\mu} \partial y^{\gamma}}=r^{\alpha-m} f_{m}(x, y) \quad(\mu+\gamma=m),
$$

where $f_{m}$ is continuous at $P$.

Proof. By [1, p. 179], $u$ is analytic in $R$. The representation $\left(27^{\prime}\right)$ below shows that the interior normal derivative $u_{n}$ is integrable on $C$. Then the analyticity of $u$ on $C$ (corners excluded) was shown by Hadamard [5, p. 25]. ${ }^{1}$

Let $t=\xi+i \eta$ and $z=x+i y$. For each $t \in R$ let $w=\Phi(z, t)$ map $R$ conformally onto the circle $|w|<1$, with $\Phi(t, t)=0$. We may assume without loss of generality that $P$ is at $z=0$, and that $\Phi(0, t)=1$. Lichtenstein [8, pp. 255-256 and footnote 273] showed" that for $m=0$, $1,2, \cdots$, and $z \in R$,

$$
\frac{\partial^{m} \Phi(z, t)}{\partial z^{m}}=z^{\alpha-m} \varphi_{m}(z, t)
$$

where $\varphi_{m}$ is continuous at $z=0$. It follows from (24) that

$$
\frac{\partial^{m} \log \Phi(z, t)}{\partial z^{m}}=z^{\alpha-m} \psi_{m}(z, t),
$$

where $\psi_{m}$ is continuous at $z=0$. Let $G(z, t)=G(\xi, \eta ; x, y)$ be Green's function for $\Delta u$ in $R$. Since

$$
G(z, t)=-(2 \pi)^{-1} \log |f(z, t)|,
$$

it follows from (25) that for $m=0,1,2, \cdots$ and $z \in R$,

$$
\frac{\partial^{m} G(z, t)}{\partial x^{\mu} \partial y^{\nu}}=r^{\alpha-m} \Psi_{m}(z, t) \quad(\mu+\nu=m),
$$

where $\Psi_{m}$ is continuous at $z=0$.

Now the function $u$ has the integral representation [1, pp. 182-183]

$$
u(x, y)=\lambda \iint_{R} G(x, y ; \xi, \eta) u(\xi, \eta) d \xi d \eta \text {. }
$$

\section{Hence}

$$
u(x+\Delta x, y)-u(x, y)
$$

1 The author wishes to thank Professor Lewy for this reference.

${ }^{2}$ Lichtenstein actually asserts that (24) is without question true for all $\alpha$, but that his proof is valid only for irrational $\alpha$. Warschawski [13] has found a simple proof of (24), valid for all $\alpha$ in the range $\frac{1}{2} \leq \alpha<\infty$.

Added in April 1954: For asymptotic expansions of $\Phi$ at a corner, see R. Sherman Lehmann, "Development of the mapping function at an analytic corner," Technical Report No. 21, Applied Mathematics and Statistics Laboratory, Stanford University, California, March 31, 1954, 17 pp. 


$$
\begin{aligned}
& =\lambda \iint_{R} \frac{G(x+\Delta x, y ; \xi, \eta)-G(x, y ; \xi, \eta)}{\Delta x} u(\xi, \eta) d \xi d \eta \\
& =\lambda \iint_{R} \frac{\partial G}{\partial x}(x+\theta \Delta x, y ; \xi, \eta) u(\xi, \eta) d \xi d \eta
\end{aligned}
$$

where $0<\theta=\theta(x, y, \Delta x)<1$. Since $G(z, t)=G(t, z)$, it is clear that $\partial G / \partial x$ $=\partial G / \partial \xi$ and, as a function of $t, \partial G / \partial x$ behaves like $\left|t-t_{0}\right|^{\alpha-1}$ at any corner $t_{0}$ of $R$, uniformly in $z$ for $z$ bounded away from $C$. Hence $(\partial G / \partial x) u(\xi, \eta)$ in (27) is dominated by an integrable function of $\xi, \eta$, uniformly with respect to $\Delta x$. By Lebesgue's convergence theorem, letting $\Delta x \rightarrow 0$ in (27) proves that

$$
\frac{\partial u}{\partial x}=\lambda \iint_{R} \frac{\partial G}{\partial x}(x, y ; \xi, \eta) u(\xi, \eta) d \xi d \eta .
$$

Setting the expression (26) for $m=\mu=1$ into the last equation proves the case $m=\mu=1$ of (23).

In a similar way one can prove all the cases $m=0,1,2,3,4$ of (23), and the lemma is established.

LEMMA 2. The functions $u_{x x}^{2}, u_{x} u_{x x x}, u u_{x x x x}, u_{y y}^{2}, u_{y} u_{y y y}$, and $u u_{y y y y}$ are Lebesgue integrable in $R$. The Lebesgue integrals $\int_{c} u_{x} u_{x x} d y$ and $\int_{C} u_{y} u_{y y} d x$ exist.

Proof. By Lemma 1 the functions $u_{x x}^{2}, \cdots, u u_{y y y y}$ are continuous in $R \cup C$ except possibly at the corners, where they are $O\left(r^{2 \alpha-4}\right)$. Since $0<\alpha$, the first sentence follows. The second sentence is proved analogously.

REMARK. The proof of Lemma 2 breaks down for corners of angle $\pi(\alpha-1)$, as $r^{-2}$ is not integrable.

Lemma 3. At any node $(x, y)$ of $R_{h}$ whose neighbors are denoted as in (2), one has

$$
\Delta^{(n)} u=\Delta u+\frac{2}{3} h\left[\theta_{x} u_{x x x}^{\prime}+\theta_{y} u_{y y y}^{\prime \prime}\right],
$$

where $-1<\theta_{x}<1,-1<\theta_{y}<1$, and where

$$
\begin{cases}u_{x x x}^{\prime}=u_{x x x}\left(x^{\prime}, y\right), & x-h_{1}<x^{\prime}<x+h_{2}, \\ u_{y y y}^{\prime \prime}=u_{y y y}\left(x, y^{\prime}\right), & y-h_{3}<y^{\prime}<y+h_{t} .\end{cases}
$$

Proof. By Lemma 1, $u_{x x x}$ is continuous in the open line segment from $\left(x-h_{1}, y\right)$ to $\left(x+h_{2}, y\right)$, but may become infinite if the endpoint is a corner of $C$. Since $u$ is continuous in $R \cup C$, it nevertheless follows 
from Taylor's formula as stated in [6, p. 357] that, if we fix $y$ and set $\phi(x)=u(x, y)$,

$$
\frac{\phi\left(x+h_{2}\right)-\phi(x)}{h_{2}}=\phi^{\prime}(x)+{ }_{2}^{h_{2}} \phi^{\prime \prime}(x)+\frac{h_{2}^{2}}{6} \phi^{\prime \prime \prime}\left(x+\theta_{2} h_{2}\right),
$$

where $0<\theta_{2}<1$.

Writing a similar formula for $h_{1}$ and subtracting, we find in the notation of (3) that

$$
D_{x}^{(h)} \phi(x)=\phi^{\prime \prime}(x)+\left[\begin{array}{c}
h_{2}^{2} \\
3
\end{array} \phi^{\prime \prime \prime}\left(x+\theta_{2} h_{2}\right)-{ }_{3}^{2} \phi^{\prime \prime \prime}\left(x-\theta_{1} h_{1}\right)\right]\left(h_{1}+h_{2}\right)^{-1} .
$$

If one writes $k=\max \left(h_{1}, h_{2}\right) \leq h$, the last term can be bounded in absolute value by

$$
\frac{2 k^{2}}{3 k} \max \left[\left|\phi^{\prime \prime \prime}\left(x+\theta_{2} h_{2}\right)\right|,\left|\phi^{\prime \prime \prime}\left(x-\theta_{1} h_{1}\right)\right|\right],
$$

and hence can be written in the form $(2 h / 3) \theta_{x} u_{x x x}^{\prime}$. Addition of a similar expression for $D_{y}^{(h)} u(x, y)$ proves the lemma.

Lemma 4. For each node $(x, y)$ of $R_{h}{ }^{2}$ defined in (15) use the notation of (28). Then, as $h \rightarrow 0$, one has

$$
h \sum_{R_{h^{2}}{ }^{2}} u\left(\left|u_{x x x}^{\prime}\right|+\left|u_{y y y}^{\prime \prime}\right|\right)=o(1) \quad(h \rightarrow 0) .
$$

Proof. The lemma is proved much like Lemma 6 of [4]. The functions $u\left|u_{x x x}\right|$ and $u\left|u_{y y y}\right|$ are continuous in $R \cup C$, except at a corner of interior angle $\pi \alpha$, where Lemma 1 states that they behave like $r^{2 \alpha-3}$ with $2 \alpha-3>-1$. The sum (29) can be majorized by the Lebesgue integral of a step function over a polygonal arc in $R$ which converges in length to $C$ as $h \rightarrow 0$. The integrability of $r^{2 \alpha-3}$ in $(0,1)$ permits the application of Lebesgue's convergence theorem as $h \rightarrow 0$. Since $u=0$ on $C$, (29) follows. Details are omitted.

Lemma 5. At each node in $R_{h}{ }^{3}$, defined in (15), one has

$$
\Delta^{(h)} u=\Delta u+\frac{1}{12} h^{2}\left(u_{x x x x}^{\prime}+u_{y y y y}^{\prime \prime}\right),
$$

where

$$
\begin{cases}u_{x x x x}^{\prime}=u_{x x x x}\left(x+\theta^{\prime} h, y\right), & -1<\theta^{\prime}<1 \\ u_{y y y y}^{\prime \prime}=u_{y y y y}\left(x, y+\theta^{\prime \prime} h\right), & -1<\theta^{\prime \prime}<1\end{cases}
$$

Proof. In [4]; the points of $R_{h}{ }^{3}$ all have four neighbors in $R_{h}{ }^{3}$, 
each at a distance $h$.

Lemma 6. At each node of $R_{h}{ }^{3}$, defined in (15), use the notation of (30). Then, as $h \rightarrow 0$, one has

$$
h^{2} \sum_{R_{h}{ }^{3}} u\left(u_{x x x x}^{\prime}+u_{y y y y}^{\prime \prime}\right)=\iint_{R} u\left(u_{x x x x}+u_{y y y y}\right) d x d y+o(1) \quad(h \rightarrow 0) .
$$

Praof. In [4].

Lemma 7. Define $u_{n}$ and $\tau$ as in Theorem 1. One then has

$$
\iint_{R} u\left(u_{x x x x}+u_{y y y y}\right) d x d y=\iint_{R}\left(u_{x x}^{2}+u_{y y}^{2}\right) d x d y+\int_{C} u_{n}^{2} \sin ^{2} 2 \tau d \tau,
$$

where the latter is a Riemann-Stieltjes integral.

Proof. The proof repeats that of Lemma 7 in [4] down to (29) of that paper. It then remains only to prove for smooth convex curves $C$ that

$$
\int_{C} u_{y y}\left(u_{y} d x+u_{x} d y\right)=\int_{C} u_{n}{ }^{2} \sin ^{2} 2 \tau d \tau \text {. }
$$

Let $s$ denote arclength on $C$, and let primes denote $d / d s$. Differentiating the relations $u_{x}=-u_{n} \sin \tau, u_{y}=u_{n} \cos \tau$, we find that, on $C$,

$$
\left\{\begin{array}{l}
u_{x}{ }^{\prime}=-u_{n}{ }^{\prime} \sin \tau-u_{n} \tau^{\prime} \cos \tau=u_{x y} \sin \tau+u_{x x} \cos \tau, \\
u_{y}{ }^{\prime}=u_{n}{ }^{\prime} \cos \tau-u_{n} \tau^{\prime} \sin \tau=u_{x y} \cos \tau+u_{y y} \sin \tau
\end{array}\right.
$$

Changing $u_{x x}$ to $-u_{y y}$ by (1), we can solve (32) for $u_{y y}$ on $C$ :

$$
u_{y y}=u_{n}{ }^{\prime} \sin 2 \tau+u_{n} \tau^{\prime} \cos 2 \tau \text {. }
$$

Since $d x=d s \cos \tau$ and $d y=d s \sin \tau$, we obtain

$$
\begin{aligned}
\int_{C} u_{y y}\left(u_{y} d x+u_{x} d y\right) & =\int_{C}\left(u_{n}{ }^{\prime} \sin 2 \tau+u_{n} \tau^{\prime} \cos 2 \tau\right)\left(u_{n} \cos 2 \tau\right) d s \\
& =\int_{C} u_{n}{ }^{2} \tau^{\prime} \cos ^{2} 2 \tau d s+\int_{C} u_{n} u_{n}{ }^{\prime} \cos 2 \tau \sin 2 \tau d s .
\end{aligned}
$$

By partial integration, we have

$$
\begin{aligned}
\int_{C} u_{n} u_{n}{ }^{\prime} \cos 2 \tau \sin 2 \tau d s & =\frac{1}{4} \int_{C}\left(u_{n}{ }^{2}\right)^{\prime} \sin 4 \tau d s \\
& =\frac{1}{4}\left[u_{n}{ }^{2} \sin 4 \tau\right]_{C}-\int_{C} u_{n}{ }^{2} \tau^{\prime} \cos 4 \tau d s .
\end{aligned}
$$

Since $\cos ^{2} 2 \tau-\cos 4 \tau \equiv \sin ^{2} 2 \tau$, substitution of (34) into (33) shows that 


$$
\int_{C} u_{y y}\left(u_{y} d x+u_{x} d y\right)=\int_{C} u_{n}{ }^{2} \tau^{\prime} \sin ^{2} 2 \tau d s
$$

Since $\tau^{\prime} d s=d \tau$, the identity (31) is proved, and with it, the lemma.

\section{REFERENCES}

1. Dorothy L. Bernstein, Existence theorems in partial differential equations, Annals of Mathematics Studies, No. 23, Princeton University Press, 1950.

2. Lothar Collatz, Eigenwertaufgaben mit technischen Anwendungen, Leipzig, Akademische Verlagsges., 1949.

3. R. Courant, K. Friedrichs, and H. Lewy, Über die partiellen Differenzengleichungen der mathematischen Physik, Math. Ann., 100 (1928), 32-74.

4. George E. Forsythe, Asymptotic lower bounds for the frequencies of certain polygonal membranes, Pac. J. Math. 4 (1954), 467-480.

5. Jacques Hadamard, Mémoire sur le problème d'analyse relatif à l'équilibre des plaques élastiques cncastrées, Mémoires Présentés par Divers Savants à l'Académie des Sciences de l'Institut National de France, sér. 2, 33, No. 4.

6. Wilfred Kaplan, Advanced calculus, Addison-Wesley, Cambridge, 1952.

7. Hans Lewy, Developments at the confuence of analytic boundary conditions, Univ. of Calif. Publications in Mathematics, new series, 1, No. 7 (1950), 247-280.

8. L. Lichtenstein, Neuere Entwicklung der Potentialtheorie, Konforme Abbildung, pp. 177-377 of Encyklopädie der mathematischen Wissenschaften, vol. 2 part 3, first half, Teubner, Leipzig, 1909-1921.

9. C. C. MacDuffee, The theory of matrices, reprint, Chelsea, New York, 1946.

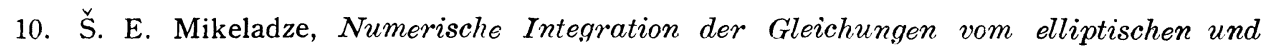
parabolischen Typus (Russian, German Summary), Izvestiya Akad. Nauk SSSR Ser. Mat. 5 (1941), 57-74.

11. D. Yu. Panov, Spravočnik po čislennomu rešeniyu differencial'nyh uravneniı $v$ častnyh proizvodnyh, 5th edit., Gostehizdat, Moscow-Leningrad, 1951.

12. G. Pólya, Estimates for eigenvalues, in Studies in mathematics and mechanics, presented to Richard von Mises, Academic Press, New York, 1954.

13. S. E. Warschawski, On a theorem of L. Lichtenstein, Pac. J. Math., 5 (1955), 835-840.

National Bureau of Standards, Los Angeles. 


\section{PACIFIC JOURNAL OF MATHEMATICS}

\section{EDITORS}

\author{
H. L. Royden \\ Stanford University \\ Stanford, California \\ E. Hewite \\ University of Washington \\ Seattle 5 , Washington
}

\author{
R. P. Dilworth
}

California Institute of Technology Pasadena 4, California

\section{A. HorN*}

University of California

Los Angeles 24, California

\section{ASSOCIATE EDITORS}

\author{
H. BUSEMANN \\ HERBERT FEDERER \\ MARSHALL HALL
}

\author{
P. R. HALMOS \\ HEINZ HOPF \\ ALFRED HORN
}

\author{
R. D. JAMES \\ BORGE JESSEN \\ PAUL LÉVY
}

GEORGE PÓLYA

J. J. STOKER

KOSAKU YOSIDA

\section{SPONSORS}

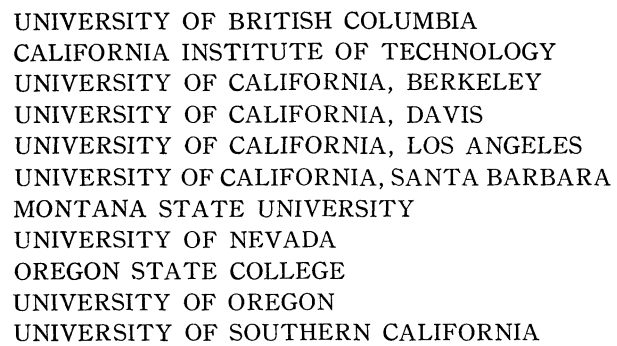

UNIVERSITY OF BRITISH COLUMBIA

CALIFORNIA INSTITUTE OF TECHNOLOGY

UNIVERSITY OF CALIFORNIA, BERKELEY

UNIVERSITY OF CALIFORNIA, DAVIS

UNIVERSITY OF CALIFORNIA, LOS ANGELES

UNIVERSITY OF CALIFORNIA, SANTA BARBARA

MONTANA STATE UNIVERSITY

UNIVERSITY OF NEVADA

OREGON STATE COLLEGE

UNIVERSITY OF OREGON

UNIVERSITY OF SOUTHERN CALIFORNIA

\author{
STANFORD RESEARCH INSTITUTE \\ STANFORD UNIVERSITY \\ UNIVERSITY OF UTAH \\ WASHINGTON STATE COLLEGE \\ UNIVERSITY OF WASHINGTON
}

AMERICAN MATHEMATICAL SOCIETY

HUGHES AIRCRAFT COMPANY SHELL DEVELOPMENT COMPANY

Mathematical papers intended for publication in the Pacific Journal of Mathematics should be typewritten (double spaced), and the author should keep a complete copy. Manuscripts may be sent to any of the editors. Manuscripts intended for the outgoing editors should be sent to their successors. All other communications to the editors should be addressed to the managing editor, Alfred Horn at the University of California Los Angeles 24, California.

50 reprints of each article are furnished free of charge; additional copies may be obtained at cost in multiples of 50 .

The Pacific Journal of Mathematics is published quarterly, in March, June, September, and December. The price per volume (4 numbers) is $\$ 12.00$; single issues, $\$ 3.50$; back numbers (Volumes $1,2,3)$ are available at $\$ 2.50$ per copy. Special price to individual faculty members of supporting institutions and to individual members of the American Mathematical Society: $\$ 4.00$ per volume; single issues, $\$ 1.25$.

Subscriptions, orders for back numbers, and changes of address should be sent to the publishers, University of California Press, Berkeley 4, California.

Printed at Kokusai Bunken Insatsusha (International Academic Printing Co., Ltd.) No. 10 1-chome Fujimi-cho, Chiyoda-ku, Tokyo, Japan.

* During the absence of E. G. Straus. 


\section{Pacific Journal of Mathematics}

\section{Vol. 5, No. $5 \quad$ BadMonth, 1955}

Henry A. Antosiewicz, A theorem on alternatives for pairs of matrice . . . . . 641

F. V. Atkinson, On second-order non-linear oscillation ............... 643

Frank Herbert Brownell, III, Fourier analysis and differentiation over real separable Hilbert spac .................................. 649

Richard Eliot Chamberlin, Remark on the averages of real function ...... 663

Philip J. Davis, On a problem in the theory of mechanical quadrature ... . . 669

Douglas Derry, On closed differentiable curves of order $n$ in $n$-spac ...... 675

Edwin E. Floyd, Boolean algebras with pathological order topologie ... . . 687

George E. Forsythe, Asymptotic lower bounds for the fundamental frequency of convex membrane ................................. 691

Israel Halperin, On the Darboux propert ................... 703

Theodore Edward Harris, On chains of infinite orde .............. 707

Peter K. Henrici, On certain series expansions involving Whittaker functions

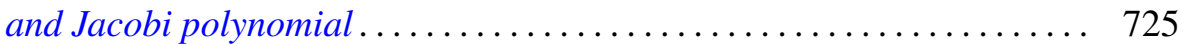

John G. Herriot, The solution of Cauchy's problem for a third-order linear hyperoblic differential equation by means of Riesz integral ......... 745

Jack Indritz, Applications of the Rayleigh Ritz method to variational

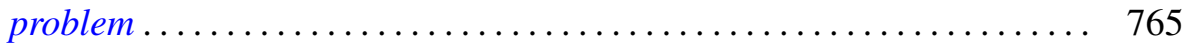

E. E. Jones, The flexure of a non-uniform bea ................. 799

Hukukane Nikaidô and Kazuo Isoda, Note on non-cooperative convex game.

Raymond Moos Redheffer and W. Wasow, On the convergence of asymptotic solutions of linear differential equation . . .

S. E. Warschawski, On a theorem of L. Lichtenstei ...........

Philip Wolfe, The strict determinateness of certain infinite game... 